\title{
Medical Image of the Week: Bronchiectasis
}

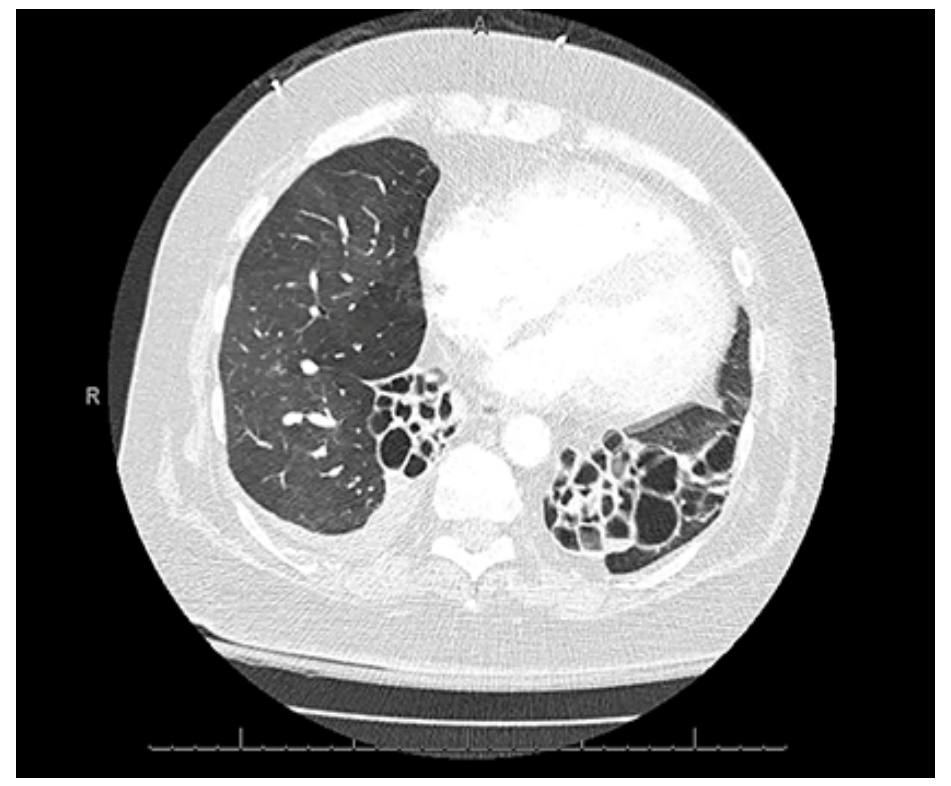

Figure 1. Thoracic axial computed tomography (CT) images in lung windows demonstrating dependent cystic bronchiectasis with air-fluid levels.

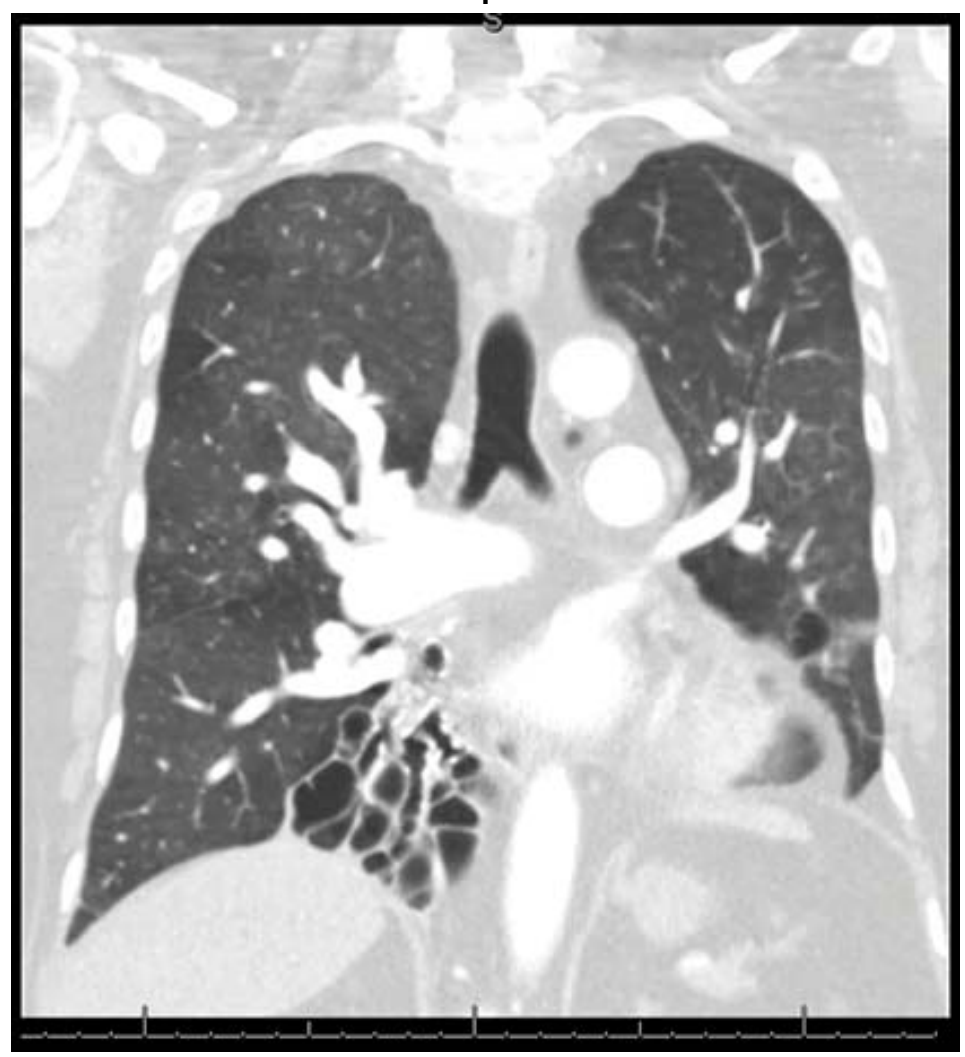

Figure 2. Thoracic coronal CT images. 
A 49-year old Native American woman with chronic hypoxic and hypercarbic respiratory failure requiring 3 liters continuous via nasal cannula and nocturnal non-invasive bi-level ventilation presented with acute shortness of breath for 5 days. She has history of recurrent respiratory infections since early childhood, however over the past five years has been treated multiple times for presumed COPD exacerbation with last such treatment one month prior to admission.

Upon arrival, vitals displayed elevated blood pressure 183/96. Clinical examination demonstrated morbidly obese patient in mild somnolence and has diffuse expiratory wheezing, basal crackles with reduced air entry bilaterally. Laboratory examination showed leukocytosis $(13,800$ cells/uL) with neutrophilic predominance, thrombocytopenia (85,000 cells/uL), and elevated bicarbonate $(31 \mathrm{mg} / \mathrm{dL})$. Arterial blood gas showed pH=7.29, pCO2 $756 \mathrm{~mm} \mathrm{Hg}$, and pO2 $73 \mathrm{~mm} \mathrm{Hg}$. Thoracic computed tomography (CT) with contrast ruled out pulmonary embolism, however demonstrated extensive cystic bronchiectasis in left upper and lower lobes, right lower lobe along with findings consistent with chronic bronchitis and bronchiolitis. (Figures 1 and 2) Bronchiectasis workup showed-low serum globulins (IgG $388 \mathrm{mg} / \mathrm{dL}$, IgM $18 \mathrm{mg} / \mathrm{dL}$, IgA $64 \mathrm{mg} / \mathrm{dL}$, with low IgG-1 $226 \mathrm{mg} / \mathrm{dL}$, IgG-2 $140 \mathrm{mg} / \mathrm{dL}$ ). Alpha Antitrypsin level was high. Blood culture, sputum culture, urine Legionella, Streptococcus pneumoniae antigen, Coccidioidomycosis serology, quantiferon and AFB stain for TB were all negative. Aggressive nebulization therapy, intermittent Bi-level positive airway pressure and antibiotics allowed her to become stabilized to a baseline oxygen requirement. She was discharged with diagnosis of acute on chronic hypoxic and hypercarbic respiratory failure secondary to flare up of severe bronchiectasis secondary to common variable immunodeficiency (CVID).

Common Variable Immunodeficiency (CVID), a subset of primary humoral immunodeficiency diseases, is a condition of inadequate immunoglobulin expression in response to antigen exposure. Prevalent equally amongst the sexes and ranges from 1 in 10,000 to 50,000 with bimodal incidence either within the first or third decade of life. Initial history is nonspecific, consisting of recurrent episodes of sinusitis and bronchitis with severity of illness dependent on level of immunoglobulin expression. The European Society for Immunodeficiency defines CVID as reduced (below 2 standard deviations of the mean) levels of $\lg G$ with reduced $\lg A$ and/or $\lg M$, together with failure to mount $a$ significant antibody response to vaccination, in the absence of a known cause. However, etiology of CVID is still incompletely understood and given the clinical heterogeneity in patient presentation, there is lack of consensus on clinical definition. Persistent sinus or respiratory complaints, in combination with finding of airway bronchiectasis lead a referral to an immunologist or pulmonologist in pursuit of diagnosis.

Bronchiectasis, a syndrome characterized by irreversible destruction, abnormal dilatation impairing clearance and leading to mucous pooling, is a common development in this impaired immune condition. Management of disease is multifactorial with symptom control, administration of appropriate immunizations and immunoglobulin replacement in agammaglobulinemia in order to curb recurrence of infections. 
Pulmonary morbidity due to bronchiectasis is common, however role of lung transplant in this patient population is unknown.

Practitioners should remain cognizant of considering CVID in patients with history of recurrent pneumonias and imaging findings of bronchiectasis to hasten specialty referral early and minimize pulmonary morbidity.

Faraz Jaffer, MD. Nirmal Singh, MD. and Jennifer Huang-Tsang, MD.

Department of Internal Medicine

University of Arizona at South Campus

Tucson, Arizona USA

\section{References}

1. Panigrahi MK. Common variable immunodeficiency disorder - An uncommon cause for bronchiectasis. Lung India. 2014 Oct;31(4):394-6. [CrossRef] [PubMed]

2. Tarzi MD, Grigoriadou S, Carr SB, Kuitert LM, Longhurst HJ.Clinical immunology review series: An approach to the management of pulmonary disease in primary antibody deficiency. Clin Exp Immunol. 2009 Feb;155(2):147-55. [CrossRef] [PubMed]

3. Cunningham-Rundles $\mathrm{C}$. How I treat common variable immune deficiency. Blood. 2010 Jul 8;116(1):7-15. [CrossRef] [PubMed] 